\title{
Utilização de Azoxistrobina no Controle da Antracnose da Mangueira
}

\author{
Rui Sales Júnior*, Frank M. da Costa, Rosiane E. M. Marinho, Glauber H. S. Nunes, Joaquim Amaro \\ Filho \& Vicente S. Miranda
}

Escola Superior de Agricultura de Mossoró, Cx. Postal 137, CEP 59625-900, Mossoró, RN, e-mail: ruisales@esam.br

(Aceito para publicação em 02/10/2003)

Autor para correspondência: Rui Sales Júnior

SALES JÚNIOR, R, COSTA, F.M., MARINHO, R.E.M., NUNES, G.H.S., AMARO FILHO, J. \& MIRANDA, V.S. Utilização de azoxistrobina no controle da antracnose da mangueira. Fitopatologia Brasileira 29:193-196. 2004.

\section{RESUMO}

A antracnose da mangueira (Mangifera indica), causada pelo fungo Colletotrichum gloeosporioides, é um dos fatores limitantes à produção de frutos sadios e comercializáveis. Conhecendo a importância desta enfermidade para a cultura, avaliou-se a eficiência do fungicida azoxistrobina em diferentes doses, comparando-as com diferentes fungicidas já utilizados no controle da antracnose da mangueira. $\mathrm{O}$ ensaio constou de dez tratamentos com quatro repetições de três plantas da cv. Tommy Atkins. Os produtos utilizados foram azoxistrobina $\mathrm{GrDa} 500 \mathrm{~g} / \mathrm{kg}\left(50 ; 75 ; 100 \mathrm{mg} . \mathrm{l}^{-1}\right.$ i.a.) acrescido de nonilfenol etoxilado a $0,05 \%$; azoxistrobina ( $75 \mathrm{mg} . \mathrm{l}^{-1}$ i.a.) acrescido de óleo mineral parafínico a 0,2 e $0,5 \%$; clorotalonil PM $825 \mathrm{~g} / \mathrm{kg}$ (1240 mg..$^{-1}$ i.a.); benomil PM 500g/kg (500 mg..$^{-1}$ i.a.); oxicloreto de cobre PM $588 \mathrm{~g} / \mathrm{kg}$ (2350 mg.1 ${ }^{-1}$ i.a.); clorotalonil/ azoxistrobina (1240/75 mg. $\mathrm{l}^{-1}$ i.a.), alternando-se esses produtos a cada pulverização, e uma testemunha sem pulverizar. Foi realizado um total de seis aplicações com intervalos de 15 dias, sendo estas iniciadas no momento da floração plena da panícula. A avaliação dos frutos foi realizada 20 dias após a última pulverização, sendo coletados 25 frutos/planta. Os mesmos foram acondicionados em caixas de papelão e armazenados a temperatura ambiente durante 15 dias. A avaliação foi realizada com base na incidência e severidade de antracnose nos frutos. O teste não-paramétrico de Kruskal-Wallis indicou que não houve diferença significativa entre os tratamentos químicos, tendo estes diferido da testemunha. Entretanto, o tratamento azoxistrobina $\left(75 \mathrm{mg} . \mathrm{l}^{-1}\right)+$ óleo mineral parafínico a $0,5 \%$ foi o que apresentou a menor incidência de frutos com antracnose.

Palavras-chave adicionais: controle químico, pós-colheita, frutos, Mangifera indica, Colletotrichum gloeosporioides.

\section{ABSTRACT}

The use of azoxystrobin in the control of mango anthracnosis

Anthracnosis is a serious disease that limits the production of healthy and marketable mangos (Mangifera indica). Due to the serious nature of this disease, a study was undertaken to test the efficacy of azoxystrobin as compared to different fungicides commonly utilized for the control of the disease in mangos. The fungicides were applied to mango plants of cv. Tommy Atkins through a ten-treatment experiment with four, three-plant replicates. The products tested were azoxystrobin WG $\left(50 ; 75 ; 100 \mathrm{mg} . \mathrm{l}^{-1}\right.$ a.i.) plus nonylphenol ethoxylate $(0.05 \%)$; azoxystrobin $\left(75 \mathrm{mg} . \mathrm{l}^{-1}\right.$ a.i.) + paraffin mineral oil (0.2 and 0,5\%), chlorothalonil WP (1240 mg..$^{-1}$ a.i.); benomyl WP (500 mg..$^{-1}$ a.i.); copper oxychloride WP (2350 mg..$^{-1}$ a.i.); chlorothalonil/azoxystrobin (1240/75 mg. $\mathrm{l}^{-1}$ a.i.), alternating these products at each application; and an unsprayed control. A total of six applications were performed at 15 days intervals, the first one performed at panicle plain flux. Fruits were evaluated 20 days after the last application, in 25 fruits/plant collections, totaling 1,000 fruits, which were conditioned in cardboard boxes and stored at ambient temperature during 15 days. The fruits were evaluated based on the incidence and severity of injury to them. The data were analyzed through the non-parametric Kruskal-Wallis test. There were no significant differences between the chemicals applied; except between these and the control. However, the treatment with azoxystrobin $\left(75 \mathrm{mg} .1^{-1}\right.$ a.i. $)+$ paraffin mineral oil in $(0.5 \%)$ was the one that exhibited the least incidence of the disease.
A cultura da mangueira (Mangifera indica L.) de ampla adaptação às condições edafoclimáticas da região Nordeste brasileira, tornou-se não só um dos cartões postais da fruticultura irrigada, mas, um dos símbolos de desenvolvimento econômico. Não obstante, muitos são os problemas encontrados na cadeia produtiva desta cultura, em muitos casos, limitantes a sua exploração comercial tendo em vista as perdas qualitativas e quantitativas produzidas nesta cultura. Um dos mais importantes é o ataque de microorganismos fitopatógenos. Entre eles, destaca-se o fungo Colletotrichum gloeosporioides (Penz.) Penz. \& Sacc. anamorfo de
Glomerella cingulata (Stoneman) Spauld. \& H. Schrenk. (Chitarra \& Chitarra, 1990; Ploetz, 1994; Cunha et al., 2000), responsável pela redução na qualidade dos frutos produzidos neste cultivo. Todos os órgãos da planta são atacados; flores, panículas, ramas, folhas e frutos (Ploetz, 1994), sendo este último afetado em qualquer estádio de desenvolvimento. $\mathrm{O}$ ataque caracteriza-se pela presença de manchas de coloração escura que ao se coalescerem aumentam de tamanho, tornando o fruto totalmente imprestável para sua comercialização, principalmente quando se trata de mercado internacional. A disseminação dos esporos de C. gloeosporioides dá-se 
principalmente pelo vento e por respingos de chuva, estando totalmente relacionado a sua incidência com a presença de molhamento foliar. Segundo Cunha et al. (1993; 2000), períodos chuvosos e encobertos como também orvalhos intensos durante o período noturno favorecem muito o desenvolvimento do patógeno. Em condições de elevada umidade relativa (superior a 90\%) (Tavares, 1995) e temperaturas superiores a 22 ?C é possível observar no centro das lesões pontuações pardo-amareladas que são as frutificações do patógeno (Kader, 1992; Ploetz, 1994; Cunha, et al., 2000).

Além de reduzir a produtividade e desqualificar comercialmente os frutos, a antracnose provoca ferimentos ou lesões nos frutos que beneficiam a infestação de fungos oportunistas e insetos (pragas), os quais podem provocar rapidamente a morte da planta ou parte desta que foi afetada (Kader, 1992; Cunha et al., 2000).

Muitos são os fungicidas recomendados em aplicações preventivas para o controle químico desta enfermidade, entre eles os cúpricos, à base de mancozebe e triazóis, tiofanato metílico a $0,05 \%$, tebuconazole a $0,25 \%$ e benomil a $0,03 \%$ (Cunha et al., 2000).

O presente trabalho objetivou avaliar a eficiência de diferentes doses de azoxistrobina, em aplicações preventivas, comparando-as com diferentes produtos já utilizados no controle da antracnose da mangueira.

$\mathrm{O}$ experimento foi conduzido em um plantio do cv. Tommy Atkins com plantas com aproximadamente 13 anos de idade, no município de Ipanguassu-RN. As mesmas apresentavam um espaçamento de plantio de $12 \times 10 \mathrm{~m}$, com uma densidade de 83 plantas/ha. O delineamento experimental utilizado foi em blocos casualizados com dez tratamentos e quatro repetições de três plantas para cada tratamento. Os tratamentos constituíram-se dos fungicidas: azoxistrobina GrDa $500 \mathrm{~g} / \mathrm{kg}\left(\right.$ Amistar $\left.^{\circledR}\right)$ nas doses de (50; 75 e $100 \mathrm{mg} . \mathrm{l}^{-1}$ i.a.) acrescidos do espalhante adesivo nonilfenol etoxilado (NE) $\left(\right.$ Fixade $^{\circledR}$ ) a 0,05\%; azoxistrobina PM (75 mg.l $\mathrm{l}^{-1}$ i.a.) acrescidos de óleo mineral parafínico (OM) $\left(\right.$ Nimbus $\left.^{\circledR}\right)$ a 0,2 e $0,5 \%$; clorotalonil PM $825 \mathrm{~g} / \mathrm{kg}$ (1240 mg..$^{-1}$ i.a.) (Bravonil ${ }^{\circledR}$ Ultrex); oxicloreto de cobre PM $588 \mathrm{~g} / \mathrm{kg}\left(2350 \mathrm{mg} . \mathrm{l}^{-1}\right.$ i.a.) $\left(\right.$ Reconil $\left.^{\circledR}\right)$; benomil PM 500 g/kg (500 mg.. ${ }^{-1}$ i.a.) (Benlate ${ }^{\circledR}$ 500); clorotalonil/azoxistrobina PM/PM (1240/75 mg..$^{-1}$ i.a.), alternando os produtos a cada pulverização e uma testemunha sem pulverizar. As pulverizações, num total de seis, foram efetuadas quinzenalmente, mediante a utilização de um equipamento de pulverização, acoplado a um trator, com duas pistolas munidas com bicos tipo Tweenjet TJ 60 de leque duplo, com jatos dirigidos. As mesmas tiveram início no dia 30 de janeiro de 2001, coincidindo com o início da floração no pomar e a última delas a aproximadamente 20 dias antes da colheita. Todas foram efetuadas em primeira marcha reduzida e, realizadas no período da manhã, dentro das condições climáticas ideais para a pulverização: UR> $60 \%$ e velocidade do vento $<10 \mathrm{Km} / \mathrm{h}$. O volume de calda preparado para cada tratamento foi de 1000 l.ha $^{-1}$, sendo utilizada uma média de $12,051^{-1}$ por planta. Um total de $265 \mathrm{~mm}$ de precipitação pluvial incidiu sobre a parcela experimental, distribuindo-se entre os meses de janeiro $(6,5 \mathrm{~mm})$, fevereiro $(0,0 \mathrm{~mm})$, março $(160,5 \mathrm{~mm})$ e abril $(98,0 \mathrm{~mm})$.

Aos 24 dias do mês de abril foi efetuada a colheita, sendo colhidos um total de 1.000 frutos, 25 por repetição. Os mesmos foram colhidos 20 dias após a última aplicação dos tratamentos fungicidas e, acondicionados em caixas de papelão tipo + exportação sem receber tratamento pós-colheita e armazenadas em condição ambiental à temperatura média diária de $31^{\circ} \mathrm{C}$ e umidade relativa ao redor dos $90 \%$, por um período de 15 dias. A distribuição das caixas obedeceu a um delineamento estatístico inteiramente casualizado. Foram avaliadas: severidade e incidência de antracnose nos frutos. Para a variável severidade adotou-se uma escala diagramática própria, atribuindo-se notas aos sintomas: 1.: ausência de manchas de antracnose nos frutos; $2 .:$ menos de $5,0 \%$ da área dos frutos afetados com antracnose; 3.: de 5,0-10,0\% da área dos frutos afetados com antracnose; $4 .:$ de 10,1 - 30\% da área dos frutos afetados com antracnose e 5.: de 30,1\% da área dos frutos afetados com antracnose.

A incidência foi obtida através do número de frutos afetados por repetição, sendo esses valores expressados em porcentagem por tratamento.

Em todos os frutos que desenvolveram sintomas de antracnose foram realizados isolamentos das manchas em meio batata-dextrose-ágar (BDA) e observados em microscópico trilocular Leica CME equipado com objetiva $\mathrm{E}_{2}$ planacromática de 40x, para confirmar a presença de $C$. gloeosporioides

Todos os dados foram analisados estatisticamente mediante a utilização do Teste não-paramétrico de "KruskalWallis", utilizando-se o programa estatístico Statistical Analisys System -"SAS".

A variável incidência de antracnose apresentou valores médios, conforme tabela 1 , variando entre 5,0 e $100 \%$. No entanto, os tratamentos $2,3,4,5,7$ e 8 apresentaram valores inferiores aos $10 \%$ de incidência do total de frutos amostrados (Figura 1). Ao contrario, os tratamentos 1, 6 e 9 apresentaram maior incidência de frutos infectados com 12, 23 e 18\%, respectivamente (Figura 1). A testemunha apresentou $100 \%$ dos frutos afetados pela antracnose (Tabela 1). A análise não paramétrica dos dados de incidência apontaram diferenças significativas para os tratamentos $2,4,5$ e 8 em relação a testemunha (tratamento 10).

É importante ressaltar que todos os frutos de manga avaliados neste ensaio foram colhidos sem a presença de sintomas da enfermidade. Isso indica que o estádio de colheita não permitiu o desenvolvimento da doença, permanecendo os esporos do fungo em dormência até o período de maturação do fruto, quando se pode apreciar o desenvolvimento das manchas provocadas por este (Cunha et al., 2000).

Houve diferença significativa entre os tratamentos com base na severidade de antracnose. A menor severidade média ocorreu no tratamento 5 (12,5\%) (Tabela 1), não diferindo estatisticamente dos demais tratamentos onde aplicou-se fungicida. 

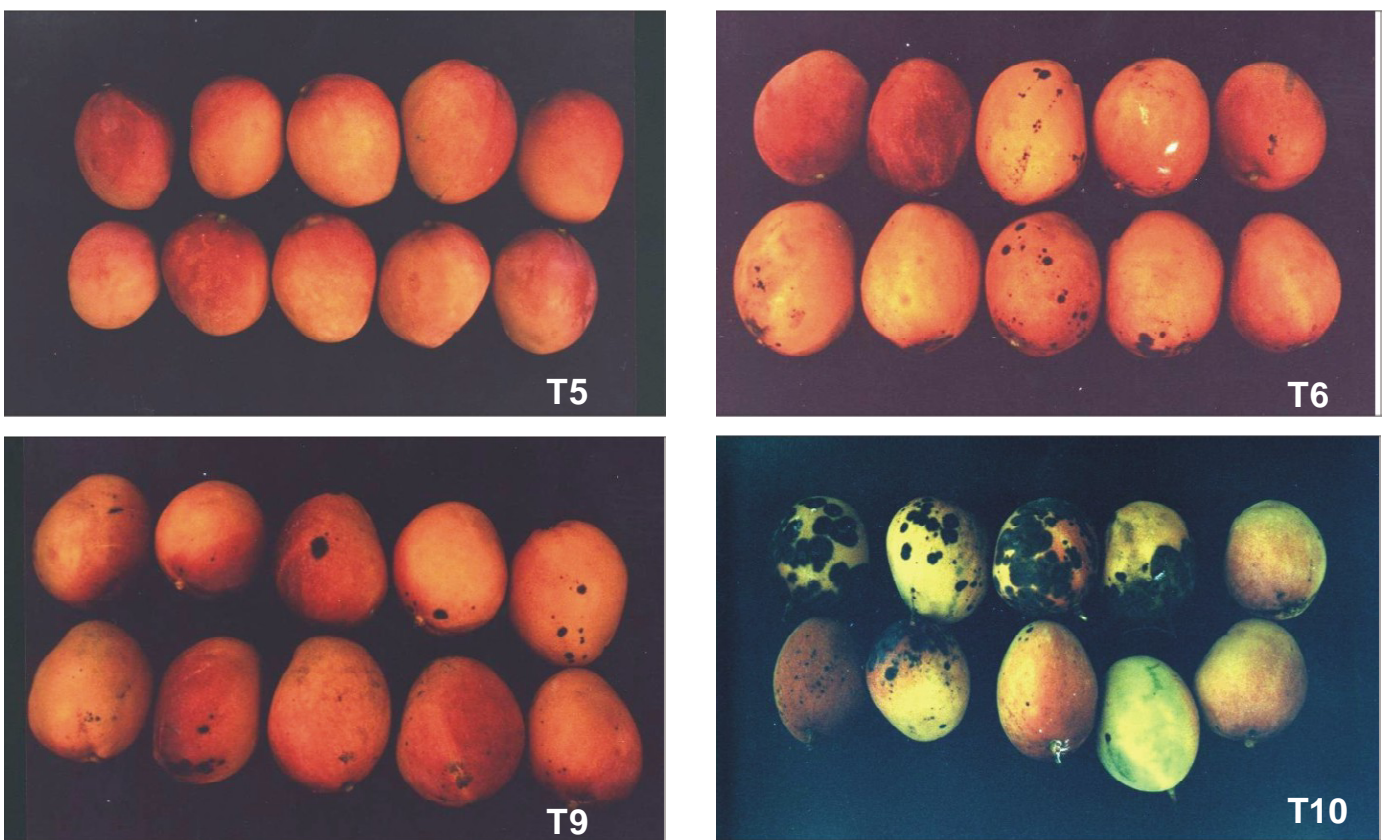

FIG. 3 - Detalhe de frutos de manga (Mangifera indica) com sintomas de antracnose após o tratamento com fungicidas no campo e armazenamento dos frutos (T5 - azoxistrobina $75 \mathrm{mg} . \mathrm{l}^{-1}+\mathrm{OM} 0,5 \%$, T6 - Clorotalonil $1240 \mathrm{mg} . \mathrm{l}^{-1}$, T9 - clorotalonil $1240 \mathrm{mg} \cdot \mathrm{l}^{-1 /}$ azoxistrobina $75 \mathrm{mg} \cdot \mathrm{l}^{-1}+\mathrm{NE}$ 0,05\% e T10 - testemunha).

Em ensaio semelhante, realizado em Petrolina-PE, Moreira et al. (2002) encontraram que os frutos tratados com azoxistrobina foram aqueles que apresentaram a menor incidência de antracnose.

Os valores obtidos na avaliação deste ensaio indicam que no período chuvoso a incidência de antracnose pode chegar a $100 \%$, sendo de notória importância à aplicação de um tratamento químico pré-colheita para o seu controle. $\mathrm{O}$ que vem a confirmar com Dood et al. (1991), sobre o efeito positivo da alta umidade relativa sobre a infecção de $C$. gloeosporioides em frutos de manga. Ao contrario, Oliveira
(2002) não detectou infecção dos frutos para todos os tratamentos aplicados em ensaio similar ao apresentado, no período seco do ano, corroborando assim sobre a importância da umidade no desenvolvimento da enfermidade.

Vale destacar que a incidência de frutos infetados poderia ser próxima de zero, caso houvesse sido realizado um tratamento pós-colheita, principalmente naqueles tratamentos que apresentaram uma incidência inferior a $10 \%$.

Spalding et al (1986) tratando frutos de manga da variedade Tommy Atkins, com imazalil, e mantendo os frutos a 24 ?C por um período de até 20 dias, obteve uma porcentagem

TABELA 1 - Efeito dos fungicidas na incidência e na severidade da antracnose em frutos de manga (Mangifera indica), após o armazenamento dos frutos

\begin{tabular}{|c|c|c|c|c|}
\hline $\mathbf{N}^{0}$ & Tratamento & $\begin{array}{c}\text { Dose ia. } \\
\left(\mathrm{mgl}^{-1}\right)\end{array}$ & Incidência (\%) e Rank & Severidade \\
\hline 1 & Azoxistrobina $+\mathrm{NE}^{1} 0,05 \%$ & 50 & $12,0\left(19,25 \mathbf{a b}^{3}\right)$ & $17,0 \mathbf{b}$ \\
\hline 2 & Azoxistrobina $+\mathrm{NE} \quad 0,05 \%$ & 75 & $6,0(15,13 \mathbf{b})$ & $21,0 \mathbf{a b}$ \\
\hline 3 & Azoxistrobina $+\mathrm{NE} \quad 0,05 \%$ & 100 & $8,0(16,63 \mathbf{a b})$ & $17,0 \mathrm{~b}$ \\
\hline 4 & Azoxistrobina $+\mathrm{OM}^{2} 0,2 \%$ & 75 & $5,0(12,75 \mathbf{b})$ & $17,0 \mathrm{~b}$ \\
\hline 5 & Azoxistrobina + OM $0,5 \%$ & 75 & $6,0(13,25 \mathbf{b})$ & $12,5 \mathrm{~b}$ \\
\hline 6 & Clorotalonil & 1240 & $23,0(29,88 \mathbf{a b})$ & $26,0 \mathbf{a b}$ \\
\hline 7 & Oxicloreto de Cobre & 2350 & $8,0(17,38 \mathbf{a b})$ & $17,0 \mathbf{b}$ \\
\hline 8 & Benomil & 500 & $8,0(15,50 \mathbf{b})$ & $17,0 \mathbf{b}$ \\
\hline 9 & Clorotalonil /Azoxistrobina + NE $0,05 \%$ & $1240 / 75$ & $18,0(26,75 \mathbf{a b})$ & $21,5 \mathbf{a b}$ \\
\hline \multirow[t]{2}{*}{10} & Testemunha & --- & $100(38,50$ a) & $38,5 \mathbf{a}$ \\
\hline & $\chi^{2}$ & & $19,264^{4}$ & 17,57 \\
\hline
\end{tabular}

${ }^{1}$ espalhante adesivo nonilfenol etoxilado

${ }^{2}$ óleo mineral parafínico

${ }^{3}$ Médias seguidas pela mesma letra não diferem entre si pelo teste de comparações múltiplas

baseado no "teste não-paramétrico de Kruskal-Wallis".

${ }^{4}$ Significativos a $5 \%$ de probabilidade (Teste de Kruskal-Wallis). 


\section{R. Sales Júnior et al.}

de frutos com sintomas de antracnose próximo a 4,0\%.

Cabe aqui ressaltar que os embarques de manga para os Estados Unidos, provenientes da região onde foi realizado este ensaio, foram comprometidos em sua totalidade na chegada aquele País, devido a presença de antracnose, já que o produtor onde foi realizado o experimento não efetuava um tratamento pré-colheita. Tendo em vista que os frutos quando são colhidos não apresentam indícios da enfermidade, este realizou apenas um tratamento hidrotérmico, exigência do mercado norte-americano, seguido de um tratamento fungicida pós-colheita, mediante imersão dos frutos, como forma de controlar a enfermidade. Dessa forma os tratamentos com azoxistrobina nas dosagens de 75 e 100 mg..$^{-1}$ i.a. acrescidos do espalhante adesivo nonilfenol etoxilado a $0,05 \%$ e azoxistrobina (75 mg. $1^{-1}$ i.a.) acrescidos de óleo mineral parafínico a 0,2 e $0,5 \%$, podem ser usados como alternativa eficiente de controle da antracnose em frutos de mangueira, sempre que acompanhados de um tratamento pós-colheita.

\section{REFERÊNCIAS BIBLIOGRÁFICAS}

CHITARRA, M.I.F. \& CHITARRA, A.B. Pós-colheita de frutos e hortaliças: Fisiologia e manuseio. Lavras, MG: ESAL/FAEP, 1990.

CUNHA, M.M., SANTOS FILHO, H.P. \& NASCIMENTO, A.S. do. Manga. Fitossanidade. Brasilia: EMBRAPA Mandioca e Fruticultura, Cruz das Almas-BA.Brasília - EMBRAPA Comunicação para Transferência de Tecnologia, Frutas do Brasil, 6,2000

CUNHA, M.M., COUTINHO, C. de C., JUNQUEIRA, N.T.V. \& FERREIRA, F.R. Manga para exportação: aspectos fitossanitários.
Ministério da Agricultura, do Abastecimento e da Reforma Agrária, Secretaria de Desenvolvimento Rural, Programa de Apoio à Produção e Exportação de Frutas, Hortaliças, Flores e Plantas Ornamentais - EMBRAPA-SPI: Brasília-DF, 1993.

DOOD, J.C., ESTRADA, A.B., MATCHAM, J. \& JEGER M.J. The effect of climatic factors on Colletotrichum gloeosporioides, causal agent of mango anthracnose, in the Philippines. Plant Pathology 40:568-575. 1991.

KADER, A.A. Postharvest Technology of Horticultural Crops. California-USA: University of California. 1992.

MOREIRA, W.A., LOPES, D.B. \& BARBOSA, F.R. Eficiência de fungicidas no controle de fungos que depreciam a qualidade de frutos de manga. Fitopatologia Brasileira 27:S222. 2002. (Resumo).

OLIVEIRA, F.M.S. Eficiência de produtos fungicidas no controle da antracnose da mangueira (Mangifera indica L.). (Monografia). Mossoró. Escola Superior de Agricultura de Mossoró. 2002.

PATEL, R.B., GOSWAMI, N.P. Post harvest diseases of mango fruits (Mangifera indica) and their control. Pathogenic Diseases 24:140-141. 1984.

PLOETZ, R.C. Mango Diseases Caused by Fungi: Antracnose. In: Ploetz, R. C., Zentmeyer, G.A., Nishijima, N.T., Rohrbasch, K.G. \& Ohr, H.D. (Eds.). Compendium of Tropical Fruit Diseases. APS Press. St. Paul, Minnesota - USA, 1994. pp.35-36.

SPALDING, D.H. \& FREEDER, W.F. Decay and Acceptability of Mangos Treated with Combinations of Hot Water, Imazalil, and gRadiation. Plant Disease 70:1149-1151. 1986.

TAVARES, S.C.C. DE H. Principais doenças e alternativas de controle. In: EMBRAPA (CPATSA, Petrolina-PE). Informações Técnicas sobre a Cultura da manga no Semi-árido Brasileiro. Brasília: EMBRAPA-SPI,Cap. V, pp.123-156. 1995. 